\title{
Patient Centered Medical Homes: Are They the Right Choice?
}

Yasir S Alsalamah ${ }^{1 *}$

${ }^{1}$ MSN, RN, Department of Nursing, Critical Care, King Abdulaziz Medical City, Ministry of National Guard-Health Affairs, POB 22490, Riyadh, 11426, Saudi Arabia

DOI: $10.36348 /$ sjnhc.2021.v04i03.006 $\quad$ | Received: 05.03.2021 | Accepted: 25.03.2021 | Published: 28.03 .2021

*Corresponding author: Yasir S Alsalamah

\section{Abstract}

Patient-Centered Medical Homes (PCMH) is a patient-centric healthcare model that puts the patient's satisfaction and overall well-being in the first place. It establishes a strong connection between the patient, nursing staff, and provider with the Primary Care Physician (PCP) leading the task force. This is important as it helps carry out a wholesome diagnosis considering all abnormal bodily activities, rather than treating only a few symptoms that arise time by time. Coordination between the patient and the physician, alongside all correlated healthcare personnel, can significantly reduce costs in the long run while also preserving better fitness and mental health. The three most prominent purposes the PCMH model serves are: a. Improving the quality of healthcare services. b. Forming strong patient relationships. c. Reducing costs by expanding delivery and access options (American Academy of Actuaries, 2014) [1].

Keywords: Patient-Centered Medical Homes (PCMH), patient-centric healthcare, patient's satisfaction, Primary Care Physician (PCP).

Copyright (C) 2021 The Author(s): This is an open-access article distributed under the terms of the Creative Commons Attribution 4.0 International License (CC BY-NC 4.0) which permits unrestricted use, distribution, and reproduction in any medium for non-commercial use provided the original author and source are credited.

\section{Patient-Centered Medical Homes (PCMH) Healthcare Model}

A PCMH model uses clinical tools to prescribe evidence-based medicines and acquire professional advice from medical consultants. PCMH emerges as the best model for patients suffering from chronic diseases, helping them save a great deal on regular check-ups and get proven medical aid.

There are several advantages and disadvantages of this model. However, it is significant to mention that its pros exceed its cons which is one reason I chose PCMH over others.

\section{Pros:}

1. A PCMH model does not generalize facilities. It offers personal care plans to thoroughly analyze the patient's condition so that no part of his/her treatment remains overlooked.

2. Such a model is entirely empirical as it monitors chronic conditions and calculates risk to predict (and suggest) future treatment options by establishing and extrapolating trends.

3. One of the best things about PCMH is that it serves as the best model to help the patient feel better. Medical consultants are often expected to work overtime, prescribe medicines online, and overbook appointments - all to ensure that the patient remains satisfied.

4. It makes effective use of IT tools such as evidence-based medicine tools, population health management tools, etc.

5. To create a better patient experience, surplus human resources are also added by carefully balancing expenses and earnings. This non-clinical staff helps build a home-like environment for the patient.

6. FFS payments often attack the pockets essentially, so not many people in the world have access to quality healthcare. On the other hand, permember-per-month (PMPM) payments are a tad less and offer more money value relatively.

7. Data reporting and analytics are used to determine quality measures, financial outcomes, and health risks of a particular patient.

8. Based on actuarial experiences and principles, budget and care coordination fees are ascertained. These budgets also aid in measuring the model's performance and effectiveness. 
Yasir S Alsalamah., Saudi J Nurs Health Care, Mar, 2021; 4(3): 84-85

Cons:

1. A PCMH model is often expensive to establish since it requires many resources to be acquired initially. Although these resources pay off in the long run, an initial investment to kick-start the program is essential.

2. Clinical and Non-clinical staff are employees of an 'organization' and are not self-employed, so they often leave almost immediately when they get a better job opportunity, hence leaving the structure un-stabilized weak.

3. Although the model has the word 'Home' in its name, it does not entirely fit what an individual would expect from his/her home. A medical home fails to provide a tension-free, cozy ambiance despite all customization and tech-tools.

4. Faulty communication has sometimes also emerged as a major con of this model, sometimes completely missing out on care coordination.

As aforementioned, the PCMH is arguably the best exchange for one's hard-earned money in better healthcare and has more benefits than negatives.

PCMH model keeps the health of the patient at the core and dedicates all resources to the quality of service. This model does not rely on a single physicians' experience to diagnose and treat a disease or malfunction. It instead forms a network of all professionals working in the healthcare market where the best come forth to offer aid suited to their expertise first to diagnose and later treat a chronic (and/or acute) disease. To achieve more accurate patient outcomes, the model incorporates into the loop online tools and electronic equipment. These PROs take in information directly from the patient by asking a set of questions. For further corroboration, the primary physician then confirms the symptoms in person and uses analytics to determine the problem. Once that is done, tech-tools are exploited to prescribe medicines considering their financial status and disease severity. Health management tool assists in thoroughly monitoring the patients' condition and taking preventive or corrective action when/if needed. Such a system rids the patient of total reliance on either technology or doctors but proposes an effective combination of the two to yield accurate outcomes (American Academy of Actuaries, 2014) [1].

PCMHs are often connected via online portals, blogs, and websites where medical help can instantly be availed at the click of a button. A global network of well-trained, experienced humans can figure out a solution for 'one problem; the problem gets solved quite easily and quickly. However, sustaining care coordination is not as easy as it seems. A few families fall through the cracks at times and suffer, especially when switches from another healthcare model are made to PCMH. The effects, though only temporary, can lead to troubles. Research has shown that providers, payers, and patients are all better connected and served in a PCMH model. Health disparities are mitigated, emergency visits are lowered, and healthcare costs are fairly reduced. The formerly used PCMH model as designed in 2008 has now been redesigned in 2017 to yield better results. The new version incorporates feedback from practice staff, doctors and PCMH certified experts developing a 'control system that can be improved over time. The redesigned model will also happen to eliminate work replication and enhance value-based services (American Academy of Actuaries, 2014) [1].

It can be concluded that while an open system may be well suited for acute disease and symptombased treatment, a long-term, inexpensive, and handy model that surpasses other healthcare models on many levels is PCMH. It sustains patient care coordination and helps create a chain of clinical members who engage interactively to play their part in making this world a better place for stay.

\section{REFERENCES}

1. New Models of Care Delivery. American Academy of Actuaries, 3-5. Issued: April 2014 